\title{
Scenes from the life of Picasso's Still Life (1922): history, materials, and conservation
}

\author{
Allison Langley $^{1} \cdot$ Kimberley Muir $^{1}\left[\right.$ [ $\cdot$ Ken Sutherland ${ }^{1}$
}

Received: 5 February 2019 / Accepted: 23 June 2020 / Published online: 21 July 2020

(c) Springer Nature Switzerland AG 2020

\begin{abstract}
Pablo Picasso manipulated his paints to achieve a range of visual effects in his painting Still Life (Art Institute of Chicago, 1953.28). The addition of excess medium and the superposition of multiple paint layers with different drying rates contributed to localized areas of wrinkling, tenting, and lifting paint. Discolored surface coatings and overpaint from an early restoration treatment posed challenges to the understanding and conservation treatment of the work. Research and analysis were undertaken to study Picasso's process and to aid treatment decisions. X-ray and infrared imaging revealed that Picasso had originally painted a neo-classical still life on the canvas. He applied a lead-white-based priming layer over the first composition before painting the linear abstract Still Life dated February 4, 1922. The results of pyrolysis-gas chromatography-mass spectrometry analysis of paint samples and restoration coatings from past treatments helped to clarify our understanding of the painting and supplement previously published analytical results.
\end{abstract}

Keywords Pablo Picasso $\cdot$ Painting technique $\cdot$ Conservation $\cdot$ Technical imaging $\cdot$ THM-Py-GCMS · Copal

\section{Introduction}

Pablo Picasso's Still Life (Art Institute of Chicago, 1953.28) (Fig. 1) is one of a series of works from his so-called linear or late Cubist mode, which spanned from late 1921 through late 1922. He produced more than 40 linear Cubist paintings during this period, all of which employ flat color fields and lines and grids of various widths. Still Life was originally owned by Gertrude Stein, a friend and collector of the artist and an important patron of modern art in early-twentieth-century Paris. The painting remained in the collection of her partner Alice B. Toklas until 1949 and entered the Art Institute of Chicago in 1953. Stein [1] wrote that "... during this period [Picasso's] pictures were very brilliant in color ... the cubic forms were continually being replaced by surfaces and lines, the lines were more important than anything else, they lived by and in themselves. He painted his pictures not by means of his objects, but by the lines."The painting is dated in the upper left corner in white paint: 4-2-22 (February 4, 1922) and is one of three works, all dated within a two-week period, in which a guitar dominates the center of the work with a wine bottle and a compote at either side. ${ }^{1}$

The complex, layered surface of Still Life, along with localized areas of wrinkling and lifting paint and the presence of disfiguring surface coatings and overpaint, prompted a closer evaluation of the artist's manipulation of his painting materials to better understand the intended appearance of the work and how it had altered over time. The painting was examined using X-radiography and transmitted and reflected infrared imaging. Medium analysis was carried out using pyrolysis gas

\footnotetext{
1 The other paintings are Nature morte, dated January 29, 1922 in the collection of the Centre Pompidou, Paris (AM 3166P) and Bottle, Guitar and Fruit Bowl, dated February 10, 1922 in a private collection.
}

Kimberley Muir, kmuir@artic.edu | ${ }^{1}$ The Art Institute of Chicago, 111 S. Michigan Ave, Chicago, IL 60603, USA. 


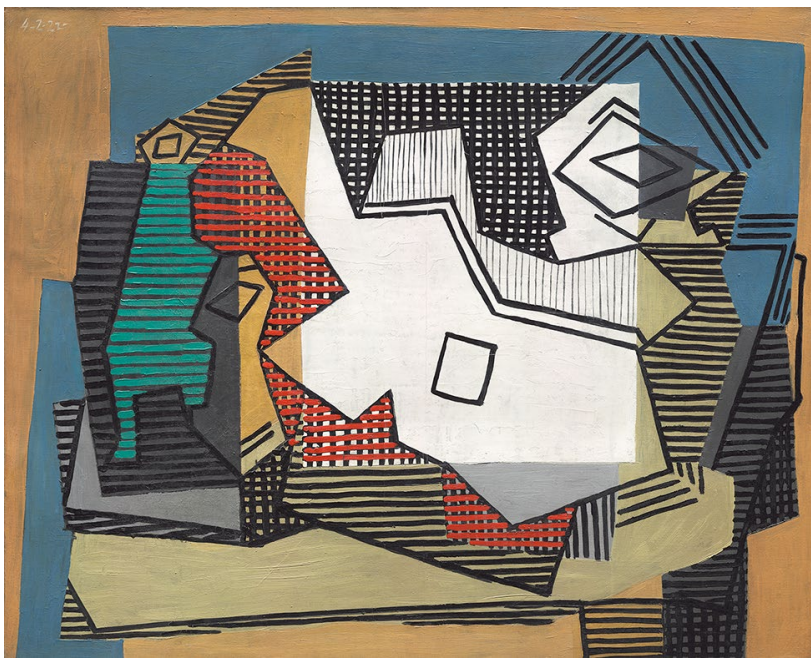

Fig. 1 Pablo Picasso, Still Life, February 4, 1922. Oil on canvas $(81.6 \times 100.3 \mathrm{~cm})$. Ada Turnbull Hertle Endowment. The image shows the painting after the most recent treatment was completed. (c) of the reproductions of works by Pablo Picasso: Pablo Picasso's Estate. VEGAP. Madrid, 2020. Permission to reproduce courtesy of VEGAP

chromatography-mass spectrometry with thermallyassisted hydrolysis and methylation (THM-Py-GCMS) to augment previous pigment and medium analysis using Fourier transform infrared spectroscopy (FTIR), scanning electron microscopy with energy dispersive $x$-ray spectroscopy (SEM-EDS), and Raman spectroscopy [2].

\section{An abandoned composition}

The painting support is a fine, plain weave canvas. It has never been lined and retains its original stretcher, which bears a brown paper label from the color merchant Bourgeois that indicates it is a no. 40 figure standard-size stretcher. The canvas appears to have been cut and prepared by the artist. Excess fabric was pulled around to the back of the stretcher, and the ground, which is present only on the image plane, was probably applied after the canvas was stretched.

From the back of the painting, it is evident that Picasso had initially started an entirely different composition on the canvas, oriented at 90 degrees to the final image. Infrared imaging of the back of the canvas helps to clarify some details of the first composition (Fig. 2). The subject is a neoclassical domestic interior still life with a pitcher, a mug, a rectangular object that may be a newspaper, and a round form mostly obscured by the vertical crossbar. The ornate curvilinear forms above and below suggest the scene

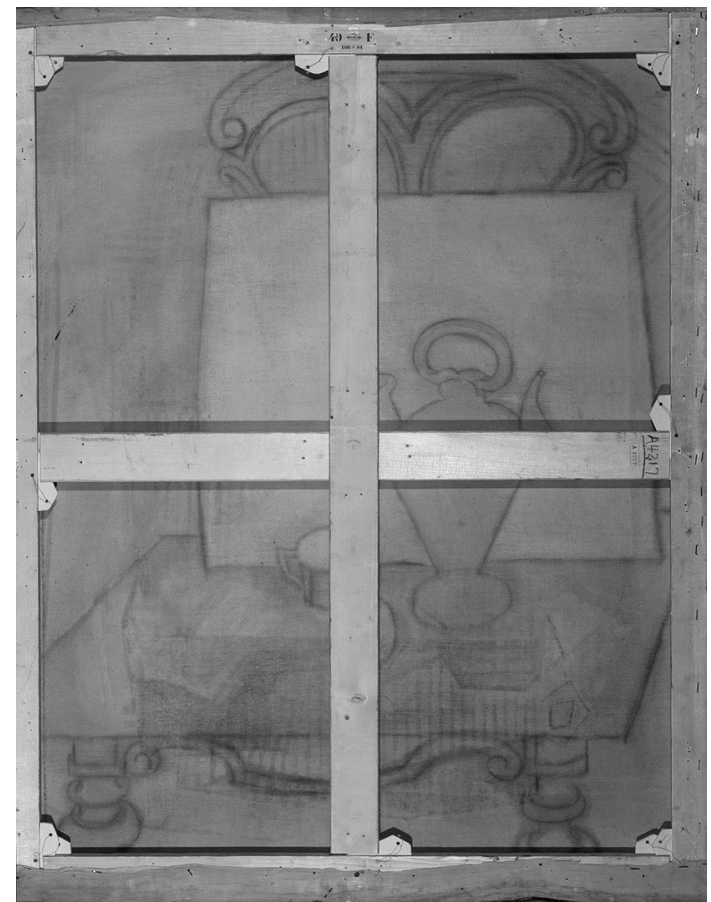

Fig. 2 Infrared image of the reverse of Picasso's Still Life showing traces of the first composition: a neo-classical still life

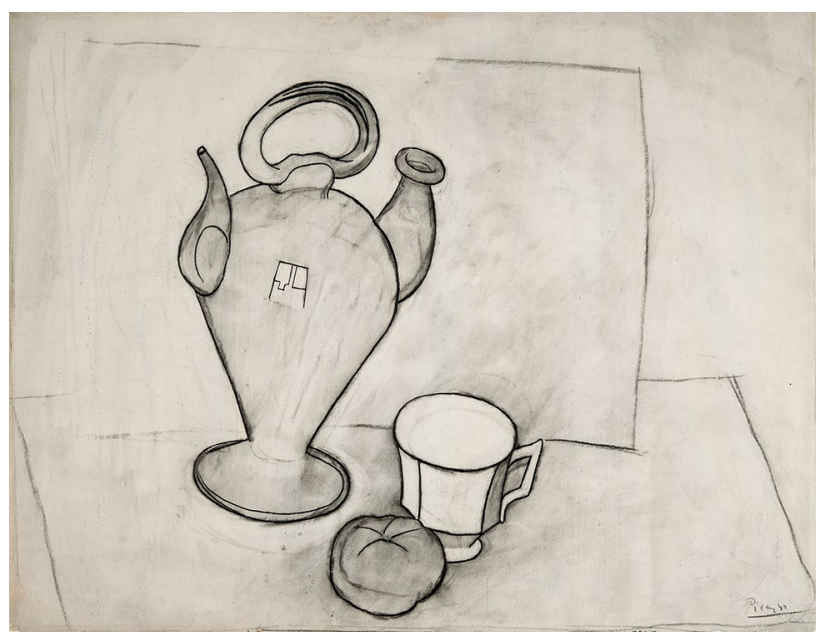

Fig. 3 Pablo Picasso, Nature morte, 1922, Gothenburg Museum of Art, Sweden (T4/1922). Gouache, pencil and crayon on paper $(46 \times 60.5 \mathrm{~cm}) \odot$ of the reproductions of works by Pablo Picasso: Pablo Picasso's Estate. VEGAP. Madrid, 2020. Permission to reproduce courtesy of VEGAP

was set up on a tabletop, or possibly a flat surface balanced on the seat of a chair; a flat rectangular object rests behind the still life objects, apparently propped against the chair back. A related drawing in the collection of the Gothenburg Museum of Art in Sweden-Nature morte, 


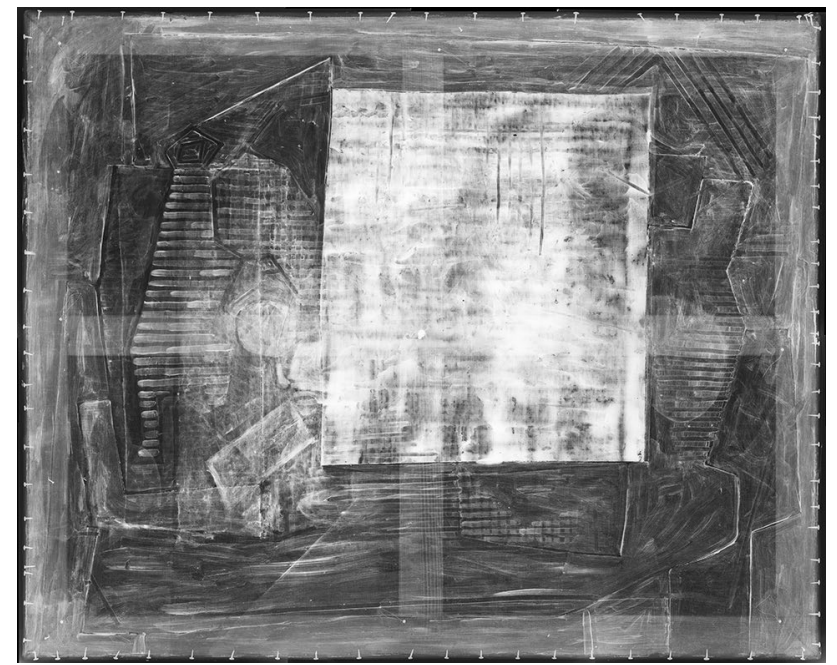

Fig. 4 X-ray of Picasso's Still Life

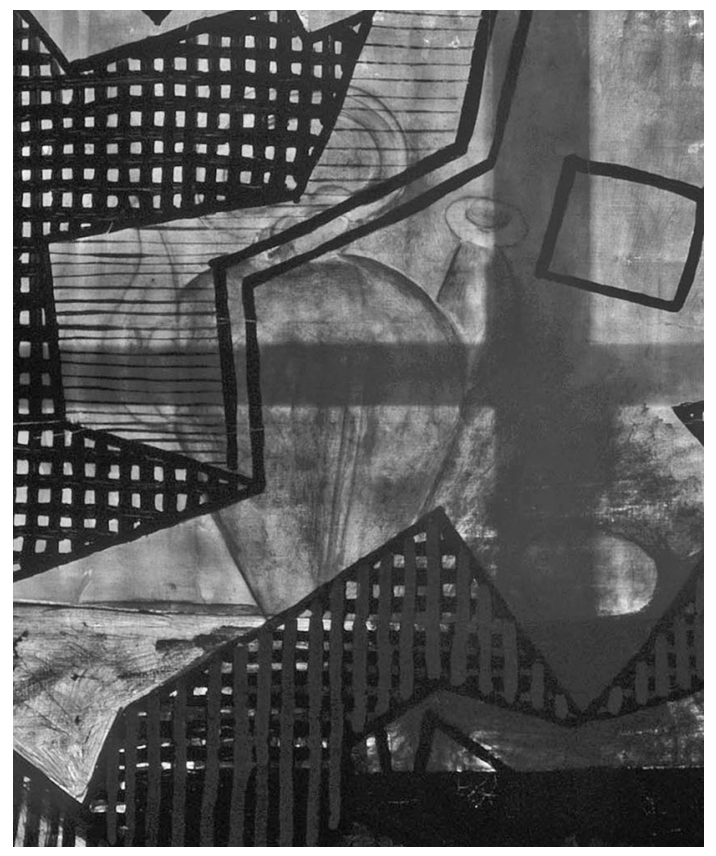

Fig. 5 Transmitted infrared detail of Picasso's Still Life showing brushwork and modeling of the pitcher from the first composition

1922-shows a similar though more distilled version of the composition and suggests that the round form in the painting may be a bread roll or a piece of fruit (Fig. 3). ${ }^{2}$ The scene was likely influenced by the interiors of Picasso's home and studio at 23 , rue la Boétie in Paris, which provided subject matter for many of his paintings and

\footnotetext{
2 We gratefully acknowledge Marilyn McCully for bringing this drawing to our attention.
}

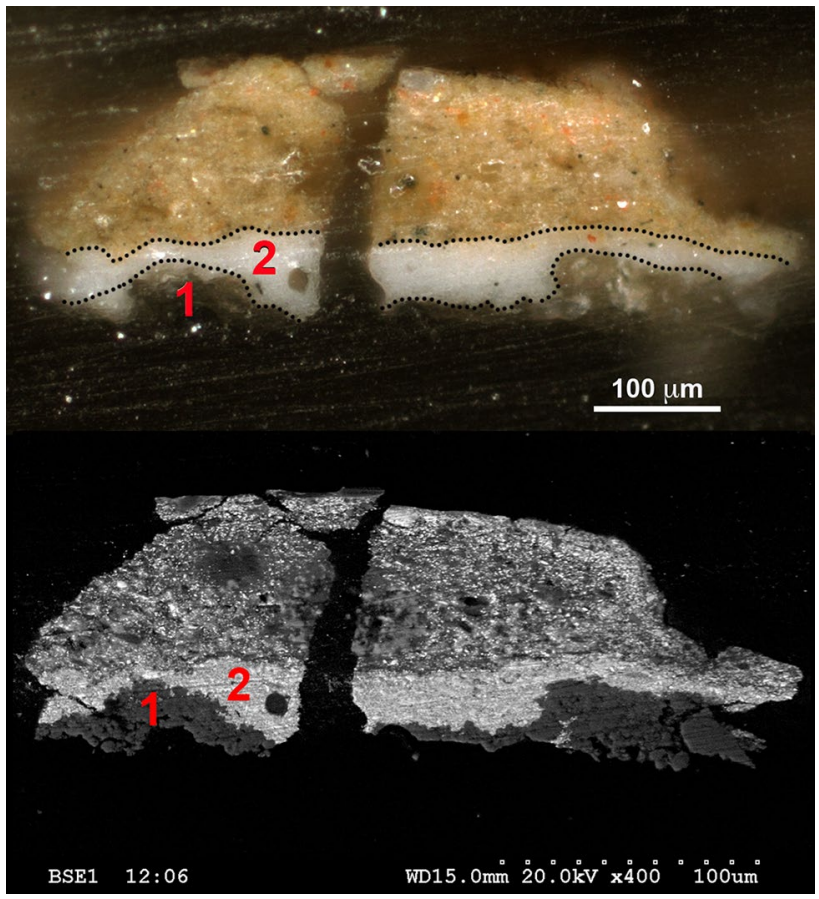

Fig. 6 Cross section of paint and ground layers from the yellow area in the upper right corner in reflected light (top) and backscattered electron image (bottom); 1 . Calcium carbonate layer. 2. Lead white layer

drawings. The composition is laid out with a line drawing executed in paint that has soaked or stained through the canvas. X-ray and transmitted infrared images indicate that parts of the composition, such as the pitcher and the smaller still life objects, were built up with some modeling of the forms (Fig. 4, 5).

A paint cross section sample from the yellow border at the upper right corner of the painting captures the artist-applied ground layer, which appears as a thin, translucent layer at the bottom of the sample in visible light and as a low-density layer in the backscattered electron image (Fig. 6). The material was identified as calcium carbonate by SEM-EDS and Raman spectroscopy [2]. The presence of this thin, absorbent layer would have allowed subsequent paint layers to soak or bleed through the ground and canvas, making the first composition visible from the back. The thick white layer in the cross section seems to be a ground layer that Picasso applied before starting the 1922 Still Life. The X-ray shows that this layer, made with lead white pigment, was applied using broad, loose brushwork and may have been used to block out the first painting and provide a uniform ground for the 1922 painting. This seems somewhat unusual in Picasso's practice, as he often painted directly over earlier compositions, allowing underlying 


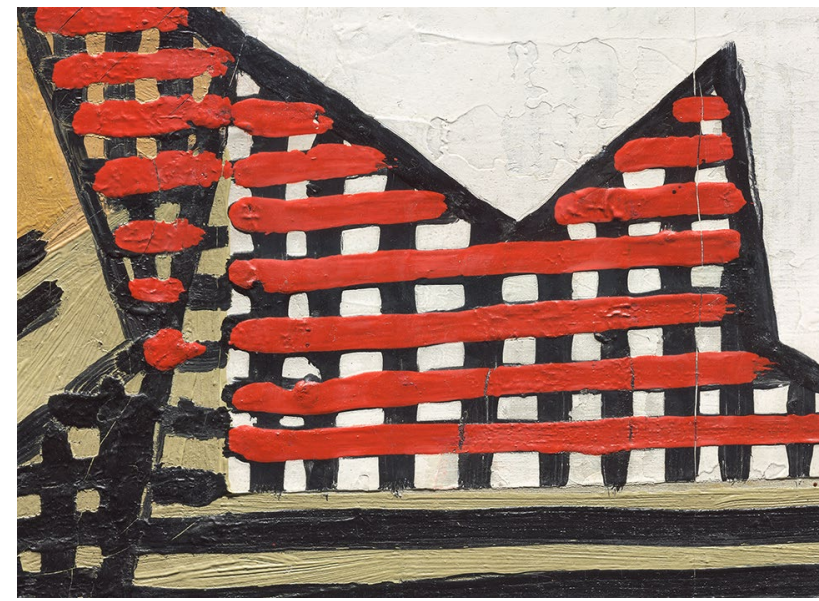

Fig. 7 Detail of Picasso's Still Life from the lower left corner of the white square, showing the variety of paint applications. (C) of the reproductions of works by Pablo Picasso: Pablo Picasso's Estate. VEGAP. Madrid, 2020. Permission to reproduce courtesy of VEGAP

forms to show through and influence the final painting [3]. The X-ray indicates that only the dense white square form near the center corresponds somewhat to an earlier form - the rectangular backdrop that leans against the back of the chair in the first painting-but the edges and overall shape have been adjusted (Fig. 4). From the surface of the painting there is no evidence of the earlier composition.

\section{Painting technique of the 1922 Still Life}

Still Life is composed of distinctly textured areas of paint built up in multiple layers (Fig. 7). Crisp brush marks define the broad areas of blue, yellow, and gray around the perimeter of the composition. For the white square in the center, Picasso used a palette knife to spread and smear the buttery paint across the surface. The red, green, and black lines creating the stripes and grids were applied with narrow flat brushes and added to the surface at a late stage in the painting process. These lines have smooth, glossier surfaces with a fluid, leveled appearance that conforms to the topography of the underlying paint layers.

On the surface, the upper paint layers often mix and run together at the edges of forms indicating that the final composition was painted in a quick campaign with little time for drying of each colored area. However, color changes observed under magnification indicate that Picasso reworked some of the broad color fields wetover-dry. The $\mathrm{x}$-ray shows that some minor shifts and adjustments of the forms were made during the painting process. However, on the whole, the color changes do not seem to correspond to actual compositional changes;

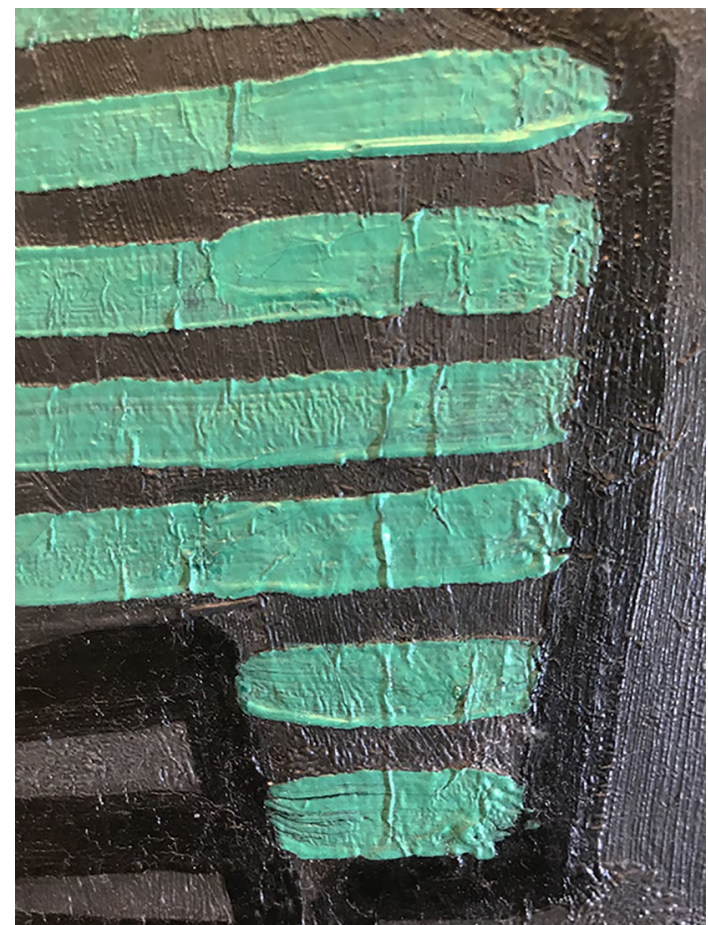

Fig. 8 Detail of Picasso's Still Life showing wrinkling and vertical ridges of tenting paint in the green stripes. () of the reproductions of works by Pablo Picasso: Pablo Picasso's Estate. VEGAP. Madrid, 2020. Permission to reproduce courtesy of VEGAP

rather they are tonal adjustments made as the artist rebalanced the overall palette of the work.

The distinct surface appearance of the red, green, and black paint, along with localized wrinkling, are qualities typically associated with the artist's use of ready-mixed, oil-based house paints such as Ripolin, which Picasso is known to have used as early as 1912 [4]. The wrinkling has occurred in some areas of the stripes but not in others and seems to have been impacted by localized differences in the build up of the paint layers and their respective drying rates. The green stripes left of center exhibit some of the most intense wrinkling, resulting in tenting and flaking paint along tiny vertical ridges (Fig. 8).

Receipts in the Picasso Archive in Paris document that Picasso purchased Ripolin in this 1921-22 period, but they also mention siccatif de Harlem. ${ }^{3}$ Although we do not know the composition of the siccative purchased by Picasso, and there were undoubtedly variations in formulation among different manufacturers and over time, historical sources indicate that it was a painting medium based on a mixture

\footnotetext{
${ }^{3}$ We are grateful to Marilyn McCully for sharing information about the 1921-22 purchase receipts in the Picasso Archives, Paris.
} 

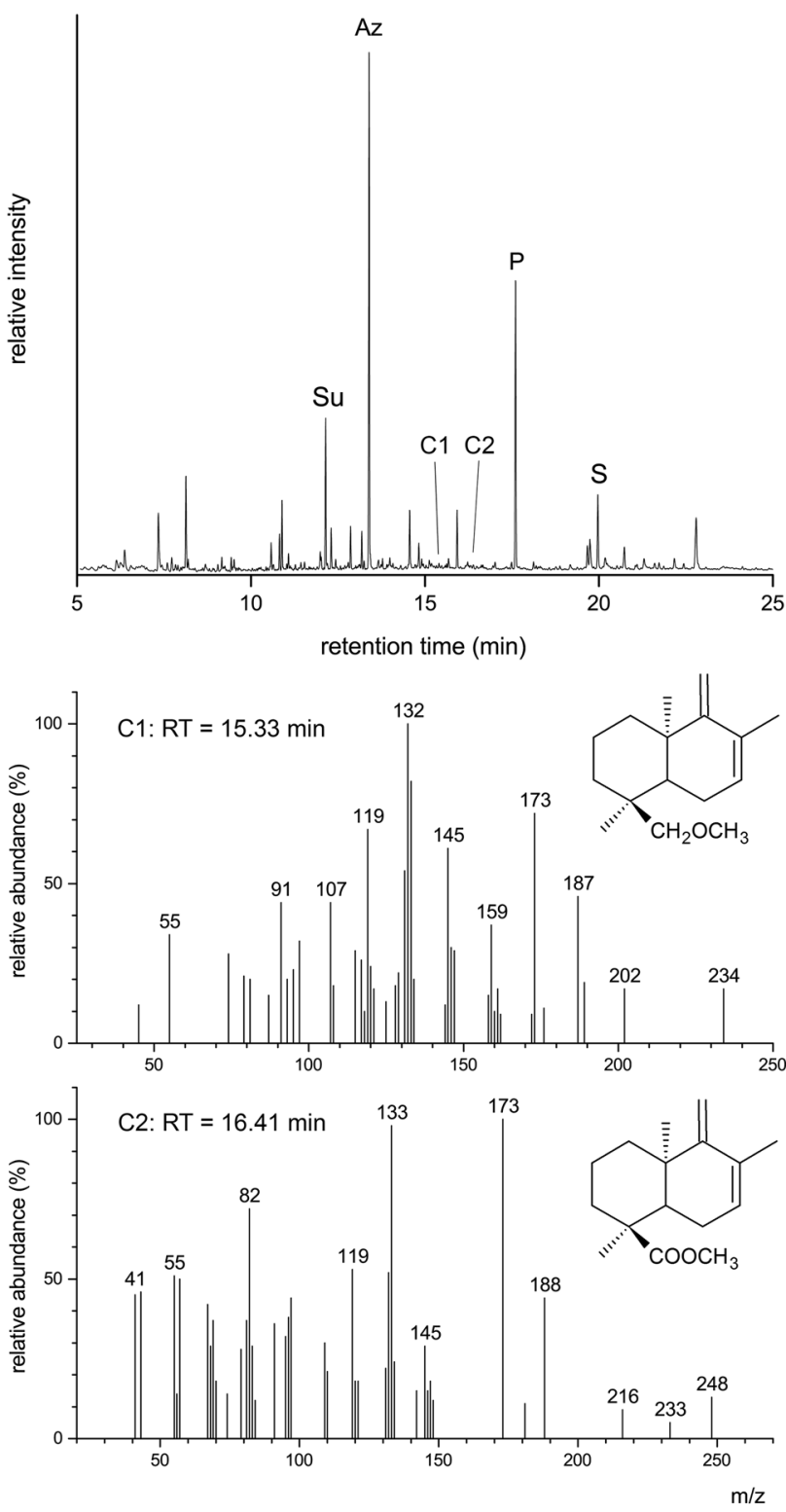

Fig. 9 Total ion chromatogram from THM-Py-GCMS analysis of glossy black paint sample (top) with mass spectra for trace pyrolysis markers, labeled $\mathrm{C} 1$ and $\mathrm{C} 2$, for copal resin. Other labeled compounds are methyl esters of suberic $(\mathrm{Su})$, azelaic $(\mathrm{Az})$, palmitic $(\mathrm{P})$ and stearic (S) acids from drying oil

of copal resin and drying oil that could impart increased body and gloss to tube paints [5].

As previously reported [2], analysis of samples from the red, green, and black paint indicated that Picasso did not use Ripolin for these areas, based on the identification of pigments such as vermilion, viridian, and lead white, and extenders such as barium sulfate, which have not been detected in extensive investigations of contemporary Ripolin paint samples [6] but are commonly used in artist's tube paints. Two samples from glossy paint areas—black

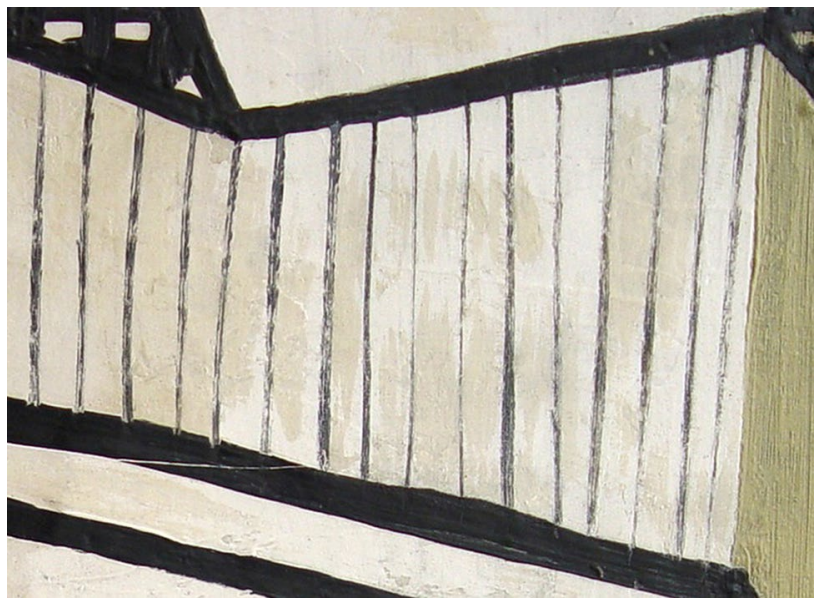

Fig. 10 Detail of Picasso's Still Life, during treatment, showing the yellowed overpaint in an area on the mid-right side of the white square. () of the reproductions of works by Pablo Picasso: Pablo Picasso's Estate. VEGAP. Madrid, 2020. Permission to reproduce courtesy of VEGAP

and red-along with samples from areas of matte black and grey paint were analyzed in the current study with THM-Py-GCMS. ${ }^{4}$ The presence of drying oil was confirmed in all samples. In addition, evidence for copal was found in the glossy black and red paint but not in the matte black and grey. The identification of copal was based on detection of trace levels of characteristic pyrolysis markers (Fig. 9; see "Appendix" for details). These findings indicate that the smooth, glossy look of some of the lines was likely achieved through Picasso's manipulation of artist's tube paint using a copal-containing medium, possibly siccatif de Harlem. In experiments mixing modern artist's tube paint with modern oil/copal painting mediums, it has been demonstrated that one can easily mimic the surface qualities of Ripolin paints, including smooth, glossy surfaces and characteristic superficial wrinkling, and that with some practice and experimentation one can begin to anticipate, if not completely control, the resulting effects [7].

\section{Conservation treatment}

Localized areas of lifting and flaking paint were consolidated with dilute BEVA ${ }^{\oplus} 371$ applied with a small brush. ${ }^{5}$ The aim was not to attempt to lay down the wrinkled and tenting paint, deemed to be an integral feature of the work, but rather to secure against any further flake losses.

\footnotetext{
${ }^{4}$ There was no remaining sample from the green paint to be used for THM-Py-GCMS analysis.

5 BEVA®371 Solution is a conservation adhesive based on ethylene vinyl acetate and cyclohexanone resins.
} 
Analysis of non-original surface coatings helped to elucidate aspects of the painting's conservation history. A discolored varnish layer from an undocumented treatment was identified by THM-Py-GCMS as an acrylic resin: poly $(n-$ butyl methacrylate). It was removed with solvents, exposing the original brighter colors and nuances of the varied paint textures and surface gloss. After cleaning, an intractable yellowed overpaint locally applied to areas of the white square became apparent (Fig. 10). The overpaint was applied over cracks in the original paint film and covered undamaged original paint. It is unclear when this restoration took place but it seems to have been conducted with an aim to even out inherent surface irregularities resulting from Picasso's palette knife application. FTIR analysis of the overpaint determined the presence of barium sulfate; THM-Py-GCMS analysis detected bleached shellac ("white lac") and drying oil..$^{6}$ The presence of shellac could account for the yellowed appearance of the overpaint, as well as its insolubility in the solvents employed for varnish removal. The overpaint was removed using a combination of solvent and mechanical action, revealing Picasso's intended surface variations. The painting was left unvarnished (see Fig. 1).

\section{Conclusions}

Picasso manipulated the handling properties and application of his paints to achieve varied surface effects and textures in Still Life. Analysis by THM-Py-GCMS supported the hypothesis that Picasso incorporated a copal-containing medium into some of the paints used for the lines and grids in order to achieve a range of smooth, fluid, and glossy surfaces. His modifications and layering of paint resulted in distinctive wrinkling patterns and ultimately insecurities in the form of tenting and lifting paint. The conservation treatment secured these fragile areas and revealed variations in surface sheen and paint application previously obscured by layers of grime, varnish, and discolored overpaint.

Acknowledgements We are grateful to Marilyn McCully, Michael Raeburn, Jennifer Poulin, Francesca Casadio, Frank Zuccari, Inge Fiedler, Maria Kokkori, Anikó Bezur, Gwenaëlle Gautier, Anna Vila, and Stephanie D'Alessandro, and for the support of the Andrew W. Mellon Foundation, the Barker Welfare Foundation, the Grainger Foundation, the Stockman Family Foundation, and the Community Associates of the Art Institute of Chicago. This work made use of the

\footnotetext{
${ }^{6}$ While "raw" or unbleached shellacs typically exhibit a bright orange fluorescence under ultraviolet (UV) illumination, the bleached resin does not possess this distinctive property (8). UV examination was therefore not helpful to investigate the distribution of the shellac-based overpaint on the surface.
}

EPIC facility of Northwestern University's NUANCE Center, which has received support from the Soft and Hybrid Nanotechnology Experimental (ShyNE) Resource (NSF ECCS-1542205); the MRSEC program (NSF DMR-1121262) at the Materials Research Center; the International Institute for Nanotechnology (IIN); the Keck Foundation; and the State of Illinois, through the IIN. Sponsorship from the Museu Picasso, Barcelona, to cover permission fees for the reproduction of works by Picasso is gratefully acknowledged.

\section{Compliance with ethical standards}

Conflict of interest The authors declare that they have no conflict of interest.

\section{Appendix:THM-Py-GCMS analysis}

Paint samples (a few micrograms) were placed in Agilent micro vials with tetramethylammonium hydroxide reagent ( $1.5 \mu \mathrm{L}$ of a $2.5 \%$ solution in methanol) in an Agilent Thermal Separation Probe, and inserted into the Multimode Inlet of an Agilent 7890B GC. The GC was equipped with an Agilent HP-5 ms Ultra Inert column ( $30 \mathrm{~m}, 0.25 \mathrm{~mm}$ i.d., $0.25 \mu \mathrm{m}$ film) and interfaced to a 5977B MS. The inlet, operated in splitless mode, was ramped from $50^{\circ} \mathrm{C}$ to $450{ }^{\circ} \mathrm{C}$ at a rate of $900^{\circ} \mathrm{C} / \mathrm{min}$ to perform THM and pyrolysis. The final temperature was held constant for $3 \mathrm{~min}$ and then decreased to $250{ }^{\circ} \mathrm{C}$ at a rate of $25^{\circ} \mathrm{C} / \mathrm{min}$. The $\mathrm{GC}$ oven was programmed from $40^{\circ} \mathrm{C}$ to $200^{\circ} \mathrm{C}$ at $10^{\circ} \mathrm{C} / \mathrm{min}$, then to $310^{\circ} \mathrm{C}$ at $6^{\circ} \mathrm{C} / \mathrm{min}$, and held isothermally for $20 \mathrm{~min}$; total run time $54.33 \mathrm{~min}$. The MS was run in scan mode ( $\mathrm{m} / \mathrm{z}$ 35-550 from 5-25 $\mathrm{min}$, and 50-700 from $25 \mathrm{~min}$ ). Drying oil was identified from the detection of a characteristic pattern of methyl esters of dicarboxylic acids (suberic, azelaic and sebacic acid) and saturated monocarboxylic acids (palmitic and stearic acid); poly ( $n$-butyl methacrylate) from detection of its $n$-BMA monomer; and bleached shellac from detection of methyl esters/ethers of characteristic hydroxy aliphatic acids and cyclic sesquiterpene acids, along with a chlorinated sesquiterpene marker [8]. Copal was indicated by the detection in the black and red paint samples of bicyclic compounds derived from pyrolysis and methylation of ozol and ozic acid polymers, designated "VIIIC" and "VIIla" by Bray and Anderson 2009 (in relation to classification of amber) [9]; see also van den Berg et al. [10]. These specific markers are characteristic for African rather than Asian copals, the latter containing polymers of communol and communic acid. They were confirmed from their retention times and mass spectra in comparison with data for known copal reference materials, published data, and spectra in NIST and Wiley databases. 


\section{References}

1. Stein $G$ (1938) Picasso. BT Batsford, London, pp 27-28

2. Muir K, Langley A, Bezur A, Casadio F, Delaney J, Gautier G (2013) Scientifically investigating Picasso's suspected use of Ripolin house paints in Still Life, 1922 and The Red Armchair, 1931. J Am Inst Conserv 52:156-172

3. Langley A, Muir K, Bezur A (2016) Looking below the surface of Picasso's The Old Guitarist. In: Wallert A (ed) Painting techniques, history, materials and studio practice, 2016 international symposium. Rijksmuseum, Amsterdam, pp 258-263

4. Leiris L, Leiris M, Kahnweiler DH, Monod-Fontaine I (1984) Donation Louise et Michel Leiris collection Kahnweiler-Leiris. Centre Georges Pompidou, Paris

5. Carlyle $L$ (2001) The artist's assistant: oil painting instruction manuals and handbooks in Britain, 1800-1900, with reference to selected eighteenth-century sources. Archetype, London, pp 48-50

6. Gautier G, Bezur A, Muir K, Casadio F, Fiedler I (2009) Chemical fingerprinting of ready-mixed house paints of relevance to artistic production in the first half of the twentieth century. I. Inorganic and organic pigments. J Appl Spectrosc 63:597-603

7. Muir K, Gautier G, Casadio F, Vila A (2011) Interdisciplinary investigation of early house paints: Picasso, Picabia and their 'Ripolin' paintings. In: Bridgland J (ed) ICOM-CC 16th triennial conference preprints, Lisbon, 19-23 September 2011. International Council of Museums, Paris, art. 1314, $10 \mathrm{pp}$

8. Sutherland K (2010) Bleached shellac picture varnishes: characterization and case studies. J Inst Conserv 33:129-145

9. Bray PS, Anderson K (2009) Identification of carboniferous ( 320 million years old) class Ic amber. Science 326(5949):132-134

10. Van den Berg K J, van der Horst J, Boon JJ (1999) Recognition of copals in aged resin/oil paints and varnishes. In: Bridgland J (ed) ICOM-CC 12th triennial meeting, Lyon, 29 August-3 September 1999, Preprints. Earthscan Ltd., London, pp 855-861

Publisher's Note Springer Nature remains neutral with regard to jurisdictional claims in published maps and institutional affiliations. 